\title{
TRANSPLANTE AUTÓLOGO DE MEDULA ÓSSEA COMO TRATAMENTO DO MIELOMA MÚLTIPLO: EXPERIÊNCIA DA UNIDADE DE TRANSPLANTE DE MEDULA ÓSSEA DO HOSPITAL PORTUGUÊS DA BAHIA
}

\author{
Autologous hematopoietic bone marrow transplantation to treat multiple myeloma: the experience of \\ the Bahia Portuguese Hospital bone marrow transplant unit
}

Ronald Pallotta ${ }^{1,2}$, Denize Ferreira Lima², Thyago Monteiro do Espírito Santo ${ }^{2}$

\begin{abstract}
RESUMO
Objetivo: Devido à distribuição heterogênea dos centros de transplante em nosso país, os autores têm por objetivo descrever a experiência de um centro nordestino de transplante de medula óssea no tratamento de mieloma múltiplo. Métodos: De setembro de 2001 a setembro de 2006 foram realizados e analisados 35 transplantes autólogos em pacientes com mieloma no Hospital Português da Bahia. Resultados: Houve predomínio do sexo feminino (1,5:1) e de não-brancos (1,3:1). A mediana de idade ao diagnóstico foi de 54 anos, sendo a maioria, secretores de IgG (68,6\%), que apresentavam estagio clínico IIIA $(88,6 \%)$. A indicação para o procedimento foi consolidação da remissão obtida inicialmente pela quimioterapia $(54,3 \%)$ ou resgate de doença refratária (45,7\%). A taxa de sobrevida global foi de $74,76 \%$, a taxa de sobrevida livre de doença foi de 60,75 \% e a taxa de mortalidade foi de 5,7\% nos primeiros 100 dias. Quando avaliamos pacientes transplantados em relação à fase da doença no momento do procedimento, observamos que os transplantados em remissão completa tiveram sobrevida livre de doença superior àqueles transplantados não em remissão (72,98\% vs 46,25\%). Conclusão: Os resultados mostraramse semelhantes aos da literatura mundial, reforçando o fato de que o transplante autólogo de medula óssea é fundamental na estratégia terapêutica contra o mieloma múltiplo e está disponível no nordeste brasileiro.
\end{abstract}

Descritores: Mieloma Múltiplo, Transplante de Células Tronco Hematopoéticas, Tratamento.

\section{Instituições:}

${ }^{1}$ Unidade de Transplante de Medula Óssea do Hospital Português da Bahia

${ }^{2}$ Núcleo de Onco-Hematologia do Departamento de Clínica Médica da Escola Bahiana de Medicina e Saúde Pública

\author{
Correspondência: \\ Dr. Ronald Pallotta \\ Av. Oceânica no 3529, 403ạ - Ondina \\ CEP 40295-050 - Salvador - BA \\ Tel: 55 71 3334-5101 \\ e-mail: tmobahia@yahoo.com.br
}

Recebido em: 06.11.2006

Aceito em: 11.01 .2007

\section{INTRODUÇÃO}

Mieloma múltiplo (MM) é uma doença maligna peculiar, que envolve não só células plasmáticas que proliferam de forma clonal, mas também o micro ambiente medular. Epidemiologicamente, essa doença, que acomete pacientes próximos da sexta década de vida é responsável por $1 \%$ de todos os tipos de câncer, e por $10 \%$ das neoplasias hematológicas. Sua incidência é de aproximadamente, 14000 casos ao ano, sendo mais freqüente em negros do que em brancos e homens são mais afetados que mulheres. ${ }^{1}$

Os enfoques terapêuticos dessa doença são extremamente complexos. A necessidade de se obter remissão completa (RC) como determinante do sucesso terapêutico é um desafio.

$\mathrm{O}$ tratamento com quimioterapia convencional resulta em baixas taxas de RC prolongada e propicia sobrevida média de três anos. Existem esquemas mais agressivos, que proporcionam maior taxa de remissão completa, mas não mostram vantagens em relação ao tratamento clássico por sua maior toxicidade e baixo impacto na sobrevida.

O transplante autólogo de medula óssea é responsável pelo 
principal avanço na terapia do MM e, apesar de não ser curativo, proporciona um aumento significativo da sobrevida global, sobrevida livre de doença e da qualidade de vida. A metade dos pacientes pode ser tratada ambulatoriamente e está associada a uma baixa taxa de mortalidade. ${ }^{2-8}$

Desta forma, tendo em vista a distribuição heterogênea dos centros de transplante no nosso país, concentrados majoritariamente nas regiões sul e sudeste, os autores descrevem a experiência de um centro no Nordeste brasileiro.

\section{OBJETIVO}

O objetivo deste estudo retrospectivo, descritivo, transversal e não controlado foi avaliar pacientes portadores de mieloma múltiplo submetidos a transplante autólogo de medula óssea no Hospital Português da Bahia, quanto à sobrevida global (SG), sobrevida livre de doença (SLD) e taxa de mortalidade relacionada ao procedimento.

\section{MÉTODOS}

De setembro de 2001 a setembro de 2006 foram realizados 35 transplantes autólogos para pacientes portadores de MM.

\section{FONTE DAS CÉLULAS}

A fonte das células-tronco hematopoéticas foi a medula óssea do próprio paciente (autólogas). Elas foram coletadas em centro cirúrgico através de múltiplas aspirações da crista ilíaca posterior, conforme a técnica universal. Foram obtidos 3 a $5 \mathrm{ml}$ de aspirado medular por punção, até um volume total equivalente a $10-15 \mathrm{ml} / \mathrm{kg}$. O material aspirado ficou em solução heparinizada até que o volume final fosse obtido. Posteriormente, ocorreu filtração da solução final e passagem para bolsas coletoras, que foram encaminhadas para congelamento.

A mediana de células coletadas foi de 3,56 x $10^{8}$ células nucleadas totais $(\mathrm{CNT})$ por quilograma de peso $(\mathrm{Kg})$ variando de 1,29 a 10,32 $\mathrm{x} 10^{8} \mathrm{CNT} / \mathrm{Kg}$, tendo sido infundido uma mediana de $3,1 \times 10^{8} \mathrm{CNT} /$ $\mathrm{Kg}$ variando de 0,96 a 9,2 x $10^{8} \mathrm{CNT} / \mathrm{Kg}$.

\section{REGIME DE CONDICIONAMENTO}

$\mathrm{O}$ condicionamento utilizado foi o melfalano na dose única de $200 \mathrm{mg} / \mathrm{m}^{2}$, tendo esta dose sido corrigida de acordo com a função renal dos pacientes, a partir da capacidade de depuração da creatinina. ${ }^{9-12}$

\section{TERAPIA DE SUPORTE}

Todo paciente admitido para transplante recebeu profilaxia parasitária com albendazol (400mg/dia por três dias) e tinidazol ( $2 \mathrm{~g} /$ dia, dose única) e profilaxia para Pneumocistis carinii com sulfametoxazol-trimetropim $(1,6 \mathrm{~g} / \mathrm{dia}$ por sete dias) no período anterior ao condicionamento.

As culturas de vigilância ( $s w a b$ nasal, oromucosa, pele, anal, perineal ou vaginal, hemoculturas e uroculturas) foram colhidas no momento da admissão.
A profilaxia para fungos foi realizada com fluconazol (400mg/ dia) e para vírus com aciclovir ( $800 \mathrm{mg} /$ dia). Elas foram instituídas no dia da infusão (dia zero) e mantidas até sete dias após a "pega medular" e até $\mathrm{D}+30$, respectivamente.

O G-CSF foi introduzido no momento em que os pacientes apresentaram contagem leucocitária inferior a 500, na dose de $10 \mathrm{mcg} / \mathrm{kg} /$ dia e mantido até obter leucometria maior que 2000 .

Os critérios para transfusão foram $\mathrm{Hb}<8,0$ e plaquetas $<20.000$ salvo casos que apresentaram sangramentos, que foram transfundidos com plaquetas $<50.000$. Todos os hemocomponentes foram administrados com irradiação prévia e com filtro leucocitário. A neutropenia febril foi tratada de acordo com o protocolo de neutropênico febril do serviço que segue as diretrizes da Infectious Disease Society of America.

\section{TERAPIA DE MANUTENÇÃO}

Nesse grupo de pacientes, todos receberam terapia de manutenção com talidomida $200 \mathrm{mg} / \mathrm{dia}$, associada a inibidor de osteólise a partir do dia +30 do transplante.

\section{ANÁLISE ESTATÍSTICA}

Foi utilizada análise estatística pelo programa GraphPad Prism v4.03 da Graphpad Software Inc. para estimar a sobrevida global, sobrevida livre de doença e mortalidade relacionada ao tratamento.

\section{RESULTADOS}

No período de seguimento dos 35 pacientes submetidos a transplante autólogo, 14 eram do sexo masculino e 21 do sexo feminino. A mediana de idade foi de 54 anos, variando entre 35 e 64 anos e a distribuição quanto à cor demonstrou $42,8 \%$ brancos e $57,2 \%$ não brancos.

Com relação à doença de base, a mediana de tempo entre o diagnóstico e o transplante foi de 22,9 meses, variando de 4,8 a 94,4 meses.

Ao diagnóstico, 68,6 \% dos pacientes eram secretores de $\mathrm{IgG}, 22,9 \%$ secretores de $\operatorname{IgA}$, e 8,6 \% não secretores. Todos apresentavam estágio clínico III, sendo $88,6 \%$ A e $11,4 \%$ B. Ao transplante, $54,3 \%$ estavam em fase de remissão completa (definida como ausência de infiltração de plasmócitos monoclonais na MO e componente M não detectável por imunofixação), 45,7\% refratários (definida como pacientes com qualquer outra resposta que não a completa). (Tabela 1).

A taxa de sobrevida global foi de $74,76 \%$ (Figura 1), a taxa de sobrevida livre de doença foi de $60,75 \%$ (Figura 2) e a taxa de mortalidade nos 100 primeiros dias foi de 5,72\%.

Quando avaliamos a sobrevida global dos pacientes transplantados em relação à fase da doença no momento do procedimento (Figura 3), observamos que aqueles submetidos à TCTH em remissão completa (RC) tiveram SG similar aos refratários RR $(79,8 \%$ vs $78,5 \%$, com $\mathrm{p}=0,7746)$. 
Tabela 1: Características dos pacientes

\begin{tabular}{lcc}
\hline & Número & $\%$ \\
\hline Pacientes & 35 & \\
Sexo & & \\
Feminino & 21 & 60 \\
Masculino & 14 & 40 \\
Cor $\quad 15$ & 42,8 \\
Branca & 15 & 52,7 \\
Não Branca & 20 & 68,6 \\
Tipo & & 22,9 \\
IgG & 24 & 08,6 \\
IgA & 08 & 88,6 \\
Não secretor & 03 & 11,4 \\
Estágio ao Diagnóstico & & \\
IIIA & 31 & 54,3 \\
IIIB & 04 & 45,7 \\
Status pré-TMo & & \\
Remissão Completa & 19 & \\
Refratário & 16 & \\
\hline
\end{tabular}

Figura 1: Sobrevida Global dos 35 pacientes submetidos à TCTH

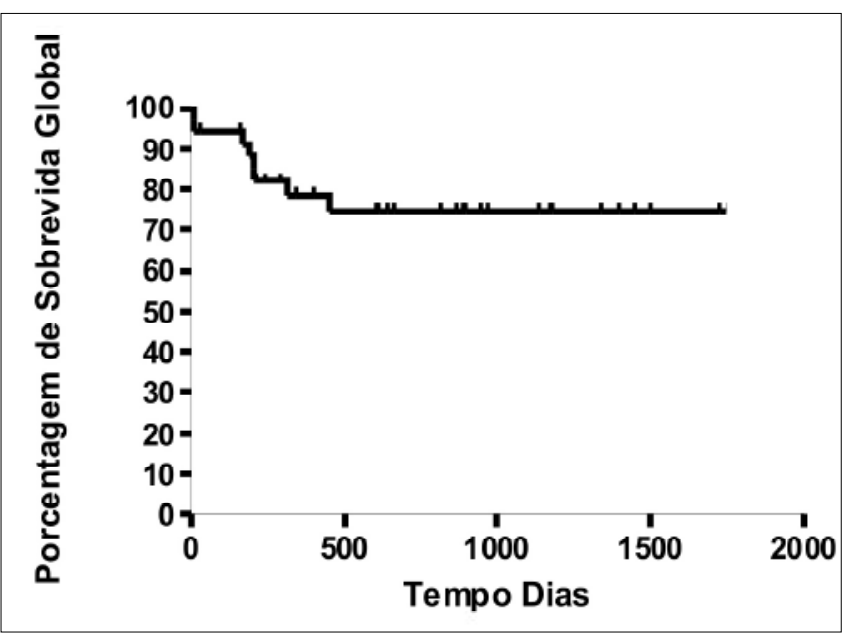

Quando comparamos a sobrevida livre de doença dos pacientes submetidos à TCTH em remissão completa (RC) com os (RR) refratários (Figura 4), observamos uma taxa superior de SLD para aqueles submetidos ao procedimento sem doença em atividade $(72,98$ vs $46,25 \%)$, embora não estatisticamente significativo $(\mathrm{p}=0,6240)$.

\section{DISCUSSÃO}

Epidemiologicamente, é possível observar na população estudada que a faixa etária no aparecimento da doença, bem como a predominância da cor não branca e prevalência do tipo de MM a partir da proteína secretada, coincide com a literatura. No entanto, a discreta predominância do sexo feminino mostra-se discrepante. ${ }^{13-25}$

Com relação aos resultados observados, encontramos que a sobrevida global $(74,76 \%)$, sobrevida livre de doença $(60,75 \%)$ e taxa de mortalidade relacionada ao procedimento $(5,72 \%)$ são compatíveis
Figura 2: Sobrevida Livre de Doença dos 35 pacientes submetidos à TCTH

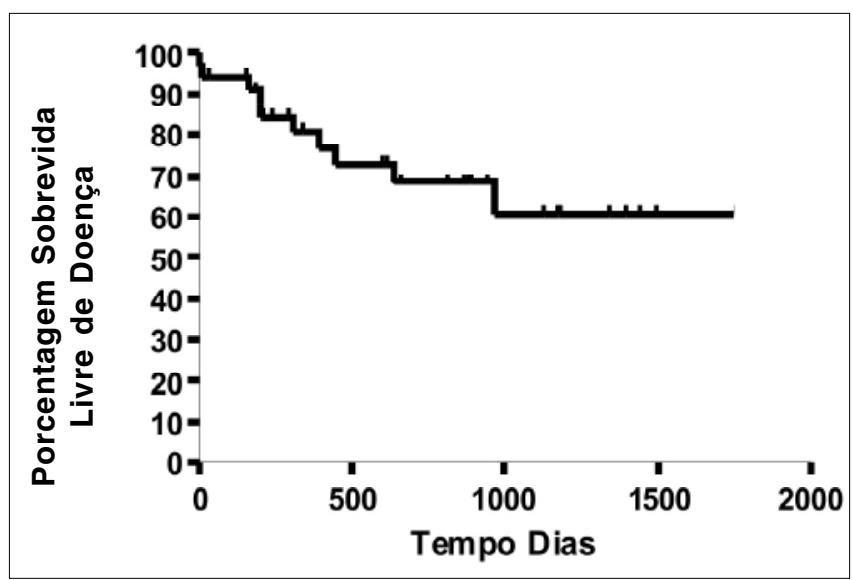

Figura 3: Sobrevida Global de acordo com a fase da doença em que os pacientes foram submetidos à TCTH

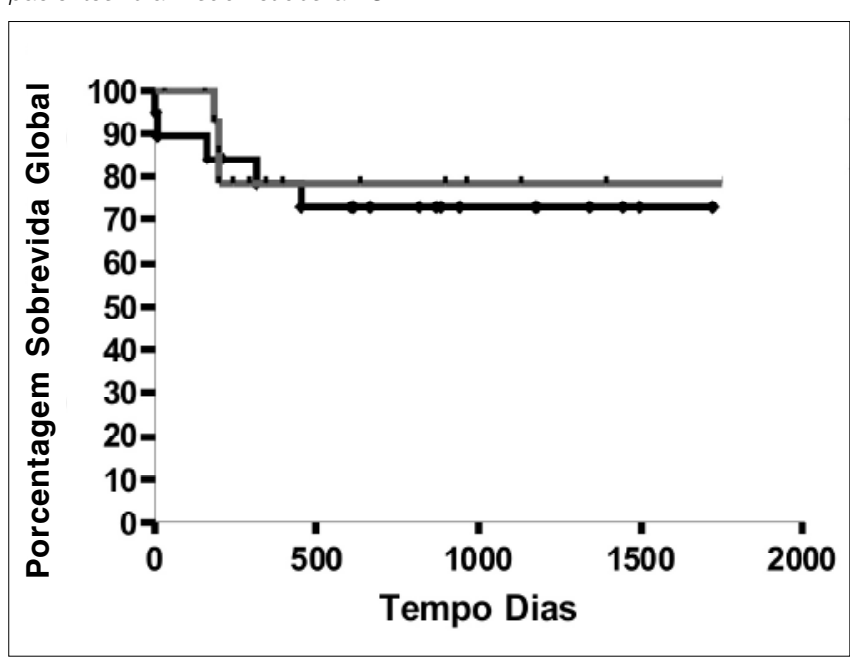

$R C=$ remissão completa, $R R=$ não remissão completa

Figura 4: Sobrevida livre de doença de acordo com a fase da doença em que os pacientes eram submetidos à TCTH

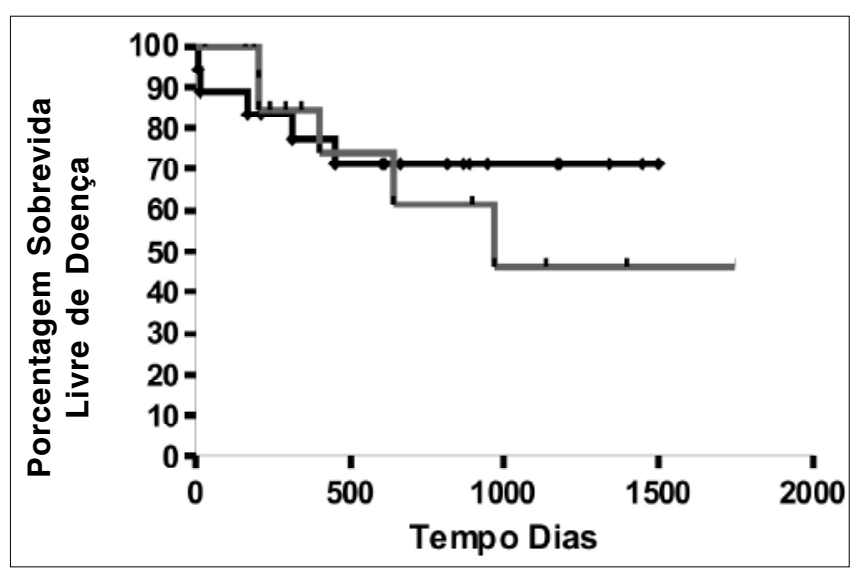

$S L D=$ sobrevida livre de doença, $R C=$ remissão completa, $R R=$ não remissão 
com os descritos na literatura mundial, mesmo o tempo mediano entre diagnóstico e procedimento tendo sido superior a 12 meses. ${ }^{13-25}$

Esse dado pode ser justificado pelo esquema de manutenção com talidomida e inibidor da osteólise precocemente introduzido e que talvez seja o responsável pelo plateau observado na curva.

Outro resultado importante relaciona-se a pacientes submetidos ao procedimento em $\mathrm{RC}$, que apresentam níveis superiores de resposta em relação aos que foram transplantados com doença em atividade no que se refere a SLD.

Esse dado, embora não estatisticamente significante em nosso estudo, está em sintonia com a lógica de quanto menor a carga tumoral, melhor o resultado. Porém, não é universalmente aceito que o grau de resposta ao esquema de quimioterapia de indução seja um fator de prognóstico para uma boa evolução no transplante. Na experiência do grupo da Mayo Clinic, por exemplo, que comparou o grau de RC após TCTH em pacientes refratários e quimiosensíveis, observou-se SG e sobrevida livres de progressão (SLP) similar nos dois grupos. ${ }^{25,26}$ Também se questiona o grau de necessidade de se atingir RC mesmo após o transplante. O mesmo grupo da Mayo Clinic demonstrou que SG e SLP foram similares em pacientes que atingiram e não atingiram RC após o TCTH..$^{25,27}$
Embora haja controvérsia quanto à resposta ao esquema de indução atuar como um fator de prognóstico, não há dúvida que mesmo pacientes refratários poderão se beneficiar do transplante. Porém, nesses casos, a manutenção é fundamental na estratégia terapêutica, e a talidomida tem se mostrado uma opção eficaz, como sugere nosso estudo. Cada vez mais, os esquemas de manutenção têm sido utilizados para maximizar os resultados com TCTH. ${ }^{25}$

$\mathrm{O}$ transplante autólogo é superior à quimioterapia convencional, principalmente no que tange à qualidade de vida. Porém, adequadas estratificações e estratégias terapêuticas devem ser traçadas a partir do diagnóstico, uma vez que o MM é uma doença heterogênea. A determinação de fatores prognósticos como o cariótipo, nível de beta 2 microglobulina, desidrogenase lática, índice de duplicação dos plasmócitos e função renal poderão adequar tal estratégia. Intervenções no regime quimioterápico de indução no transplante propriamente dito e/ou no regime de manutenção podem ser realizadas com intenção de se otimizar os resultados.

\section{CONCLUSÃO}

Os resultados mostraram-se semelhantes aos da literatura mundial, reforçando o fato de que o TCTH autólogo é fundamental na estratégia terapêutica contra o MM e está disponível no Nordeste brasileiro.

\section{ABSTRACT}

Purposes: Based on the heterogeneous distribution of transplantation centers in Brazil, the authors describe their experience treating multiple myeloma in a Northeast bone marrow transplant unit. Methods: From September 2001 to September 2006,35 autologous transplants for patients with myeloma were performed and analyzed at the Bahia's Portuguese Hospital. Results: There was a prevalence of females (1.5:1) and Caucasians (1.3:1). The median age at the diagnosis was 54, the major part IgG (68.6\%) secretes and presenting stage IIIA $(88.6 \%)$. The indication for the procedure was consolidation of the initially attained remission by chemotherapy (54.3\%) or salvage for refractory disease (45.7\%). The overall survival was of $74.76 \%$, the disease-free survival was $60.75 \%$, and the mortality rate was $5.72 \%$ in the first 100 days. Results also showed that patients in complete remission had better disease-free survival than those not in remission (72.98\% vs $46.25 \%$ ). Conclusion: It is feasible to conclude that results showed to be similar from the literature, reinforcing the fact that autologous bone marrow transplantation is fundamental in the therapeutic strategy against multiple myeloma, and it is available in the Northeast region of Brazil.

Keywords: Multiple Myeloma, Hematopoietic Stem Cell Transplantation, Treatment

\section{REFERÊNCIAS}

1. Sirohi B, Powles R. Multiple Myeloma. Lancet. 2004;363:875-87.

2. Bensinger WI, Rowley SD, Demirer T, et al. High-dose therapy followed by autologous hematopoietic stem-cell infusion for patients with multiple myeloma. $\mathrm{J}$ Clin Oncol. 1996;14:1447-56.

3. Oken MM. Multiple myeloma. Med Clin North Am. 1984;68:757-87.

4. Tricot G, Vesole DH, Jagannath S, et al. Graft-versus-myeloma effect: proof of principle. Blood. 1996;87:1196-8.

5. Verdonck LF, Lokhorst HM, Dekker AW, et al. Graft-versus-myeloma effect in two cases. Lancet. 1996;347:800-1.

6. Aschan J, Lonnqvist B, Ringden O, et al. Graft-versus-myeloma effect. Lancet. 1996;348:346
7. Bird JM, Russell NH, Samson D. Minimal residual disease after bone marrow transplantation for multiple myeloma: evidence for cure in long-term survivors. Bone Marrow Transplant. 1993;12:651-4.

8. Kyle RA, Rajkumar SV. Multiple Myeloma. N Engl Med J 2004; 351:1860-73.

9. McElwain TJ, Powles RL. High-dose intravenous melphalan for plasma-cell leukaemia and myeloma. Lancet. 1983;2:822-4.

10. Barlogie B, Alexanian R, Dicke KA, et al. High-dose chemoradiotherapy and autologous bone marrow transplantation for resistant multiple myeloma. Blood. 1987;70:869-72.

11. Phillips GL, Shepherd JD, Barnett MJ, et al. Busulfan, cyclophosphamide, and melphalan conditioning for autologous bone marrow transplantation in hematologic malignancy. J Clin Oncol. 1991;9:1880-8. 
12. Dimopoulos MA, Alexanian R, Przepiorka D, et al. Thiotepa, busulfan, and cyclophosphamide: a new preparative regimen for autologous marrow or blood stem cell transplantation in high-risk multiple myeloma. Blood. 1993;82:2324-8.

13. Attal M, Harousseau JL, Stoppa AM, et al. A prospective, randomized trial of autologous bone marrow transplantation and chemotherapy in multiple myeloma. N Engl J Med. 1996;335:91-7.

14. Attal M, Harousseau JL, Stoppa AM, et al. High-dose therapy in multiple myeloma: an updated analysis of the IFM 90 protocol. Blood. 1997;90:1858

15. Harousseau JL, Milpied N, Laporte JP, et al. Double-intensive therapy in high-risk multiple myeloma. Blood. 1992;79:2827-33.

16. Barlogie B, Jagannath S, Vesole, DH, et al. Superiority of tandem autologous transplantation over standard therapy for previously untreated multiple myeloma. Blood. 1997;89:789-93.

17. Attal M, Payen C, Facon T, et al. Single versus double transplant in myeloma: a randomized trial of the "inter groupe francais du myelome" (IFM). Blood. 1997;90:1859

18. Schiller G, Vescio R, Freytes C, et al. Transplantation of CD34+ peripheral blood progenitor cells after high-dose chemotherapy for patients with advanced multiple myeloma. Blood. 1995;86:390-7.

19. Bensinger WI. Should we purge. Bone Marrow Transplant. 1998;21:113-5.
20. Fermand JP, Brouet JC. Marrow transplantation for myeloma. Annu Rev Med. 1995;46:299-307.

21. Gahrton G, Tura S, Ljungman P, et al. Prognostic factors in allogeneic bone marrow transplantation for multiple myeloma. J Clin Oncol. 1995;13:1312-22.

22. Seiden MV, Schlossman R, Andersen J, et al. Monoclonal antibody-purged bone marrow transplantation therapy for multiple myeloma. Leuk Lymphoma. 1995;17:87-93.

23. Couban S, Stewart AK, Loach D, et al. Autologous and allogeneic transplantation for multiple myeloma at a single centre. Bone Marrow Transplant. 1997;19:783-9.

24. Bjorkstrand B, Ljungman P, Svensson H, et al. Allogeneic bone marrow transplantation versus autologous stem cell transplantation in multiple myeloma: a retrospective case-matched study from the European Group for Blood and Marrow Transplantation. Blood. 1996;88:4711-8.

25. Hari P, Pasquini MC e Vesole DH. Cure of Multiple Myeloma - more hope, less reality. Bone Marrow Transplant. 2006;37:1-18

26. Krumar S, Lacy MQ, Dispenzieri A, Rajukumar SV, Fonseca R, Geyer S, et al. High dose therapy and autologous stem cell transplantation for multiple myeloma poorly responsive to initial therapy. Bone Marrow Transplant. 2004;34:161-7

27. Rajkumar SV, Fonseca R, Dispenzieri A, Lacy MQ, Witzig TE, Lust JÁ e cols. Effect of complete response on outcomefollowing autologous stem cell transplantation for myeloma. Bone Marrow Transplantation. 2000;26:979-83 